\title{
Vortices in self-gravitating disks
}

\author{
G. R. Mamatsashvilii, ${ }^{* \dagger}$ and W. K. M. Rice** \\ *SUPA, Institute for Astronomy, University of Edinburgh, Blackford Hill, Edinburgh EH9 3HJ, \\ Scotland; E-mail:grm@roe.ac.uk \\ ${ }^{\dagger}$ Georgian National Astrophysical Observatory, Il. Chavchavadze State University, 2a Kazbegi \\ Ave., Tbilisi 0160, Georgia \\ ${ }^{* *}$ SUPA, Institute for Astronomy, University of Edinburgh, Blackford Hill, Edinburgh EH9 3HJ, \\ Scotland
}

\begin{abstract}
Vortices are believed to greatly help the formation of $\mathrm{km}$ sized planetesimals by collecting dust particles in their centers. However, vortex dynamics is commonly studied in non-selfgravitating disks. The main goal here is to examine the effects of disk self-gravity on the vortex dynamics via numerical simulations. In the self-gravitating case, when quasi-steady gravitoturbulent state is reached, vortices appear as transient structures undergoing recurring phases of formation, growth to sizes comparable to a local Jeans scale, and eventual shearing and destruction due to gravitational instability. Each phase lasts over 2-3 orbital periods. Vortices and density waves appear to be coupled implying that, in general, one should consider both vortex and density wave modes for a proper understanding of self-gravitating disk dynamics.

Our results imply that given such an irregular and rapidly changing, transient character of vortex evolution in self-gravitating disks it may be difficult for such vortices to effectively trap dust particles in their centers that is a necessary process towards planet formation.
\end{abstract}

Keywords: Hydrodynamics, Instabilities, Planetary systems: formation, protoplanetary disks PACS: $97.10 . \mathrm{Gz}$

\section{INTRODUCTION}

It is well known that antcyclonic vortices can help the planet formation process by aggregating dust particles in their centers to build planetesimals [1, 2, 3]. Numerical simulations [4, 5, 6] demonstrate that coherent anticyclonic vortices indeed emerge in disks and survive for hundreds of orbits. All these investigations are, however, carried out for non-self-gravitating disks. Here we investigate the effects of disk self-gravity on the vortex formation and evolution, because protoplanetary disks are in general selfgravitating and usually do not cool fast enough to get directly fragmented into giant planets. Instead, they settle into a quasi-steady gravitoturbulent state [7]. Hence, there should be found some mechanism that will build planetesimals in this state. Global simulations of the dynamics of dust particles in self-gravitating gaseous disks show that large scale spiral structure in a self-regulated state does concentrate dust particles in overdense/overpressure spiral arms [8]. As mentioned, another possibility of dust particle concentration is their trapping by anticyclonic vortices (worked out originally for non-self-gravitating disks). So, in perspective our present study will allow us to see if the latter mechanism of planetesimal formation can also be at work in selfgravitating disks. Due to resolution constraints, it is difficult to see vortices in global 
disk simulations. For this purpose we work in the local shearing sheet approximation.

\section{PHYSICAL MODEL AND EQUATIONS}

In the shearing sheet model only a local patch of a disk in the vicinity of some radius $r_{0}$ is considered that rotates around the central star with the angular velocity $\Omega_{0} \equiv \Omega_{K}\left(r_{0}\right)$, where $\Omega_{K}(r)$ is the angular velocity of Keplerian (differential) rotation. Within this patch the differential rotation of a disk manifests itself as a parallel shear flow with a constant velocity shear [9]. The unperturbed background surface density $\Sigma_{0}$ and twodimensional pressure $P_{0}$ corresponding to this shear flow are assumed to be spatially constant. Coriolis force is also included to take into account the effects of rotation. As a result, in this local approximation the continuity equation and equations of motion take the form [10]:

$$
\begin{gathered}
\frac{\partial \Sigma}{\partial t}+\nabla \cdot(\Sigma \mathbf{u})-q \Omega_{0} x \frac{\partial \Sigma}{\partial y}=0 \\
\frac{\partial \mathbf{u}}{\partial t}+(\mathbf{u} \cdot \nabla) \mathbf{u}-q \Omega_{0} x \frac{\partial \mathbf{u}}{\partial y}=-\frac{\nabla P}{\Sigma}-2 \Omega_{0} \hat{\mathbf{z}} \times \mathbf{u}+q \Omega_{0} u_{x} \hat{\mathbf{y}}-\nabla \psi .
\end{gathered}
$$

This set of equations is supplemented by Poisson's equation for a razor-thin disk

$$
\Delta \psi=4 \pi G\left(\Sigma-\Sigma_{0}\right) \delta(z) .
$$

Here $\mathbf{u}\left(u_{x}, u_{y}\right), P, \Sigma$ and $\psi$ are, respectively, the perturbed velocity relative to the background parallel shear flow $\mathbf{u}_{\mathbf{0}}\left(0,-q \Omega_{0} x\right)$, the two-dimensional pressure, the surface density and the gravitational potential of the gas sheet. $x$ and $y$ are, respectively, the radial and azimuthal coordinates. $\hat{\mathbf{y}}$ and $\hat{\mathbf{z}}$ are the unit vectors in the azimuthal and vertical directions, respectively. Since (1-2) are written for perturbed velocities, only the gravitational potential due to the perturbed surface density $\Sigma-\Sigma_{0}$ is used. The shear parameter $q=1.5$ for the Keplerian rotation considered here.

The equation of state is

$$
P=(\gamma-1) U,
$$

where $U$ and $\gamma$ are the two-dimensional internal energy and adiabatic index, respectively. We will adopt $\gamma=2$. The sound speed is $c_{s}^{2}=\gamma P / \Sigma=\gamma(\gamma-1) U / \Sigma$.

The central quantity of this study is the vertical component of potential vorticity referred to as PV below:

$$
I \equiv \frac{\hat{\mathbf{z}} \cdot \nabla \times \mathbf{u}+(2-q) \Omega}{\Sigma}=\frac{1}{\Sigma}\left(\frac{\partial u_{y}}{\partial x}-\frac{\partial u_{x}}{\partial y}+(2-q) \Omega\right) .
$$

The PV will play an important role in the subsequent analysis, as it generally characterizes the formation of coherent structures (vortices) in a disk flow [6].

The evolution of the internal energy density is governed by the equation

$$
\frac{\partial U}{\partial t}+\nabla \cdot(U \mathbf{u})-q \Omega_{0} x \frac{\partial U}{\partial y}=-P \nabla \cdot \mathbf{u}-\frac{U}{\tau_{c}},
$$



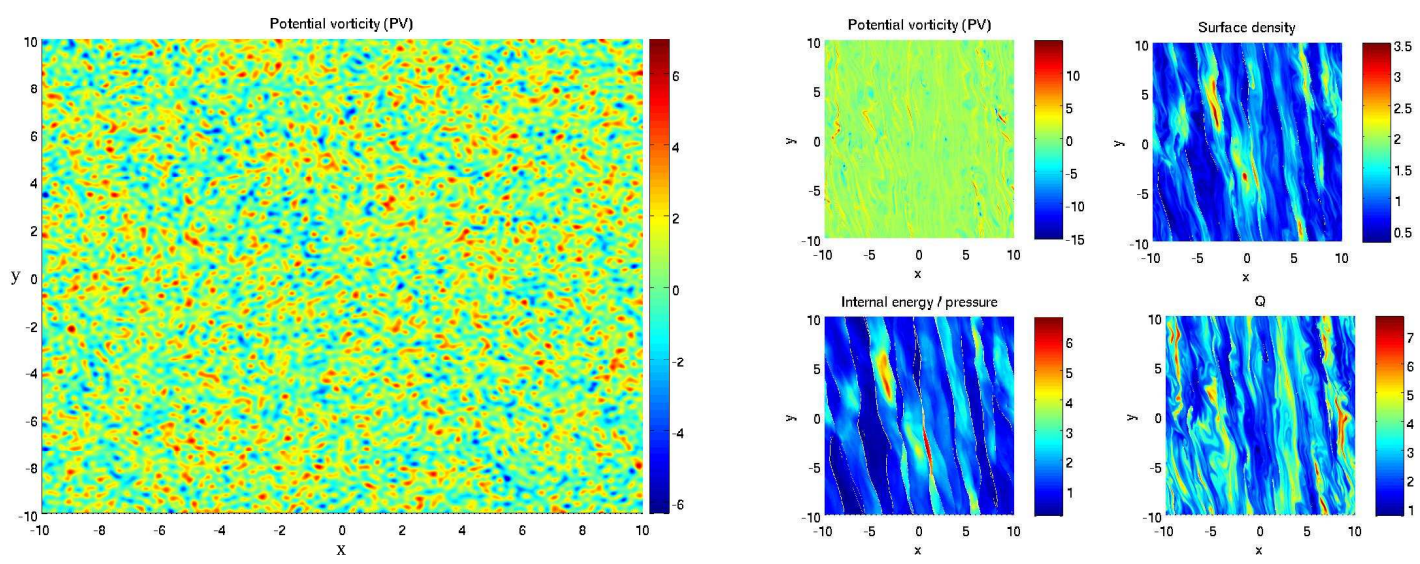

FIGURE 1. Left: Initial PV field at $t=0$ corresponding to Kolmogorov spectrum of velocities. Right four panels: PV, surface density, internal energy/pressure and Toomre's parameter $Q$ in the gravitoturbulent state at $t=33$ and $L=20$ (evolution picture remains unchanged for larger times). Adjusted negative $\mathrm{PV}$ regions produce overdense regions, which are gravitationally unstable. Unadjusted negative PV regions correspond to central underdense regions surrounded by overdense regions, though not so strong as for adjusted PV regions.

where the last term on the rhs takes account of cooling of the disk. The cooling time $\tau_{c}$ is assumed to be constant, $\tau_{c}=20 \Omega^{-1}$, so that the disk does not fragment and enters a saturated gravitoturbulent state. In the present study we concentrate on examining the peculiarities of potential vorticity evolution in such a gravitoturbulent state.

We introduce the nondimensional variables: $t \rightarrow \Omega_{0} t,(x, y) \rightarrow\left(x \Omega_{0} / c_{s 0}, y \Omega_{0} / c_{s 0}\right), \Sigma \rightarrow$ $\Sigma / \Sigma_{0}, P \rightarrow P / c_{s 0}^{2} \Sigma_{0}, U \rightarrow U / c_{s 0}^{2} \Sigma_{0}, I \rightarrow I \Sigma_{0} / \Omega_{0}$. These nondimensional variables are used throughout what follows. The Toomre's parameter is $Q=c_{s} \Omega_{0} / \pi G \Sigma$. We start with $Q=Q_{0}=c_{s 0} \Omega_{0} / \pi G \Sigma_{0}=1.5$.

Our computational domain in the $(x, y)$ plane is a square $-L / 2 \leq x, y \leq L / 2$, divided into $N \times N$ grid cells. We take $L=20$ and $N=1024$. In order to study the evolution of the system we numerically integrate (1-4) within this domain.

\section{NONLINEAR EVOLUTION}

Initial conditions consist of random $u_{x}$ and $u_{y}$ perturbations superimposed on the mean Keplerian shear flow. Surface density and internal energy are not perturbed initially. Fig. 1 shows these initial conditions in terms of PV. The velocity perturbations are measured by $\sigma=\left\langle\mathbf{u}^{2}(x, y)\right\rangle^{1 / 2}$, where the angle brackets mean ensemble averaging. In our calculations $\sigma=0.6$ at $t=0$. We start with Kolmogorov power spectrum $\left\langle|u(k)|^{2}\right\rangle \sim$ $k^{-8 / 3}$, where $k$ is the wavenumber. These random velocity perturbations are meant to mimic an initial turbulent state in a disk resulting from the collapse of a molecular cloud core.

In the presence of both Keplerian shear and self-gravity, the main mechanism responsible for the growth of initial velocity perturbations is swing amplification instead of pure Jeans instability [10, 11, 12]. During swing amplification velocity perturbations induce 
strong surface density perturbations in the form of trailing shocks with superimposed density structures. After about 4-5 orbital periods balance is reached between shock and compressional heating and cooling. As a result, the disk settles down to a quasi-steady gravitoturbulent state. The snapshot (at $t=33$ ) of the system evolution in this state is shown in fig.1. $Q$ fluctuates around 2.4, but the $Q(x, y)$ map is very inhomogeneous and contains values as small as 0.6 associated with some negative PV regions (see below). The positive (cyclonic) PV regions remain sheared into strips showing no signs of vortex formation during the entire course of evolution. Only negative (anticyclonic) PV regions are able to survive in shear flows and wrap up into more or less vortex-like structures. The overall picture of the PV evolution is still irregular and chaotic in the quasi-steady phase (fig. 1). So, we use the term 'vortex' in a broader sense meaning negative PV regions in general even if they do not have well-defined vortical shape. Some of the vortices by this time are not adjusted yet, i.e., they produce underdense regions corresponding to the centers of vortices surrounded by higher density regions related to density waves/shocks generated during the adjustment process. At the same time, we also see in this figure vortices that have already undergone adjustment phase, have grown to sizes comparable to the local Jeans scale, and correspond to stronger overdense regions. At the location of these overdense regions, $Q$ reaches small values (0.6-0.7) implying that they are gravitationally unstable and are in the process of being sheared and destroyed. (Vortex growth in size is, in general, a consequence of inverse energy cascade in 2D turbulence). During this process the temperature/internal energy rises, the corresponding region becomes stable and the vortex formation process described above starts again.

In conclusion, in self-gravitating disks the evolution of vortices has irregular and transient character in contrast to that in non-self-gravitating disks. Vortices form, undergo adjustment phase and finally appear as overdense regions in the surface density field, which afterwards become gravitationally unstable and are destroyed shortly. After that the whole process recurs.

\section{ACKNOWLEDGMENTS}

G.R.M. would like to acknowledge the financial support from the Scottish Universities Physics Alliance (SUPA). The numerical code used here was kindly provided by $\mathrm{C}$. Gammie.

\section{REFERENCES}

1. P. Barge, and J. Sommeria, A\&A, 295L, 1 (1995).

2. A. Johansen, A. Andersen, and A. Brandenburg, A\&A, 417, 361 (2004).

3. H. Klahr, and P. Bodenheimer, ApJ, 639, 432 (2006).

4. P. Godon, and M. Livio, ApJ, 523, 350 (1999).

5. O. Umurhan, and O. Regev, $A \& A, 427,855$ (2004).

6. B. Johnson, and C. Gammie, ApJ, 635, 149 (2005).

7. A. Boley, A. Mejia, R. Durisen, K. Cai, M. Pickett and P. D’Alessio, ApJ, 651, 517 (2006).

8. W. K. M. Rice, G. Lodato, J. Pringle, P. Armitage, and I. Bonnell, MNRAS, 372L, 9 (2006).

9. P. Goldreich, and D. Lynden-Bell, MNRAS, 130, 125 (1965).

10. C. Gammie, ApJ, 553, 174 (2001). 
11. W.-T. Kim, and E. Ostriker, ApJ, 559, 70 (2001).

12. G. R. Mamatsashvili, and G. D. Chagelishvili, MNRAS, 381, 809 (2007). 